\begin{tabular}{|c|l|}
\hline Title & What kind of stratospheric sudden warming propagates to the troposphere? \\
\hline Author(s) & Nakagawa, Ken I.; Y amazaki, Koji \\
\hline Citation & $\begin{array}{l}\text { Geophysical Research Letters, 33, LO4801 } \\
\text { https:/doi.org/L0.1029/2005GLO24784 }\end{array}$ \\
\hline Issue Date & 2006-02-16 \\
\hline Doc URL & http://hdl.handle.net/2115/1438 \\
\hline Type & article (author version) \\
\hline Note & $\begin{array}{l}\text { An edited version of this paper was published by A GU. } \\
\text { Copyright 2006, A merican Geophysical Union, Geophysical Research Letters, 33 }\end{array}$ \\
\hline File Information & GRL33.pdf \\
\hline
\end{tabular}

Instructions for use 


\title{
What kind of stratospheric sudden warming propagates to the troposphere?
}

\author{
Ken I. Nakagawa ${ }^{1}$, and Koji Yamazaki ${ }^{2}$
}

1 Sapporo District Meteorological Observatory, Japan Meteorological Agency

Kita-2, Nishi-18, Chuoh-ku, Sapporo, 060-0002 Japan

Tel: +81 116116124 FAX: +81 116116121

ken-ichi.nakagawa@met.kishou.go.jp

2 Faculty of Environmental Earth Science, Hokkaido University

Kita-10, Nishi-5, Kita-ku, Sapporo, 060-0810 Japan

Tel: +81 $117062361 \quad$ FAX: +81 117064865

yamazaki@ees.hokudai.ac.jp

Nakagawa, K. I and K. Yamazaki, (2006): What kind of stratospheric sudden warming propagates to the troposphere? , Geophysical Research Letters, 33, L04801, doi:10.1029/2005GL 024784. [published 16 February 2006] 


\section{Abstract}

The factors affecting the downward propagation of stratospheric sudden warming (SSW) events to the troposphere are studied through composite analysis of 45-year reanalysis data from the European Centre for Medium-Range Weather Forecasts. During the growth stage of SSW, events that propagate into the troposphere exhibit enhanced upward flux of the wavenumber 2 wave, while events that do not propagate downward display reduced wavenumber 2 flux. In both events, upward flux of the wavenumber-1 wave is enhanced, but the enhancement is stronger in the non-propagating event. The composite for propagating events reveals a negative Eurasian pattern of horizontal geopotential anomalies in the troposphere during the growth stage, and a negative Arctic Oscillation pattern following the event, while non-propagating events are preceded by a positive Eurasian pattern. In both types of event, the tropospheric anomalies are generated mainly by tropospheric planetary wave forcing prior to the emergence of SSW.

\section{Introduction}

Stratospheric sudden warming (SSW) is a sudden breakdown of the stratospheric polar vortex caused by dynamical forcing of upward-propagating planetary waves from the troposphere [Andrews et al., 1987]. Recently, a growing body of evidence has accumulated showing that changes in stratospheric circulation affect tropospheric circulation. A change in the strength of the stratospheric polar vortex propagates into the troposphere within a few weeks, and the influence of stratospheric circulation on the troposphere persists for up to two months [Baldwin and Dunkerton, 1999, 2001]. The negative phase of the Arctic Oscillation (AO) [Thompson and Wallace, 1998, 2000] in the troposphere tends to emerge after SSW events [Limpasuvan et al., 2004].

However, the negative phase of the AO, representing a period of weak tropospheric polar vortex, does not always arise after stratospheric events. For example, the December 1998 SSW event did not propagate into the troposphere, allowing a strong tropospheric vortex to form, whereas the subsequent SSW in February 1999 did 
propagate into the troposphere to induce the negative AO [Baldwin and Dunkerton, 2001]. It therefore appears useful to examine the factors that cause SSW events to propagate into the troposphere. The present paper attempts to clarify the influences on the downward propagation of SSW events through composite analysis of reanalysis data.

\section{Data and analysis}

The data set examined in the present study is the 45-year (September 1957 to August 2002) reanalysis data from the European Centre for Medium-Range Weather Forecasts [Simmons and Gibson, 2000]. Daily averages calculated from 4th daily data for the full 45-year period are adopted. Daily Eliassen-Palm (EP) fluxes [Andrews et al., 1987] are calculated as averages of the 4th daily fluxes. Daily climatological values are calculated by applying a 20-day low-pass filter to the 45-year average values. All anomalies presented in this study denote deviations from the daily climatological values.

The occurrence of an SSW event in this study is determined based on the polar temperature, which is defined as the area-mean temperature poleward of $80^{\circ} \mathrm{N}$. The first criterion of an SSW event is a polar temperature warming rate greater than $20 \mathrm{~K}$ per 6-day period at 10, 20 or $30 \mathrm{hPa}$. If the warming only satisfies the criterion for one day at $10 \mathrm{hPa}$, the warming is discarded. A key-day is defined as the day on which the warming rate is maximized at $10 \mathrm{hPa}$. The strength of an SSW event is defined as the maximum temperature rise at $10 \mathrm{hPa}$ over 6 days centered around the key-day. When two SSW events occur with an interval of less than 30 days, the stronger SSW event is selected and the weaker event is discarded. Based on these criteria, a total of 51 SSW events were finally selected for analysis. The 51 SSW events examined here include weaker SSWs compared with the 39 events selected by Limpasuvan et al. [2004] from the 44-year data. However, composites prepared for the subset of strong SSW events (polar temperature rise greater than $35 \mathrm{~K}$ over 6 days, 17 events) yield similar results to those presented below.

The 51 SSW events are classified into 2 groups based on the propagation of 
stratospheric polar temperature anomalies into the troposphere, as determined from 30-day averaged anomalies in polar temperature at 500 hPa (PT500). Events involving a positive 30-day mean anomaly for PT500 after the key-day are classified as tropospheric warm events (28 events), and those associated with a negative anomaly are designated as tropospheric cold events (23 events).

\section{Results}

The polar night jet is decelerated in association with an SSW event. Figure 1 shows the composite anomalies of zonal mean high-latitude $\left(50-80^{\circ} \mathrm{N}\right)$ zonal wind from day -40 to day +40 for tropospheric warm and cold events. Prior to both types of events (before day -5), preconditioned positive anomalies occur in the stratosphere followed by a rapid weakening of the zonal wind around the key-day to the anomaly minimum at day +5 . In both types of events, downward propagation of the negative anomaly occurs in the stratosphere after the SSW, followed by gradual recovery from the upper stratosphere. The features of SSWs in the middle and upper stratosphere are common in both cases.

The differences between the two types of events are most apparent below $100 \mathrm{hPa}$. The composite anomalies of zonal mean high-latitude zonal wind at $300 \mathrm{hPa}$ are shown in Figure 2a for both events. In the composite for tropospheric warm events, negative anomalies appear around day -7 and persist beyond day +40 . In the tropospheric cold events, on the other hand, the positive anomaly extends from day -8 to day +28 , and the stratospheric negative anomaly does not propagate below $150 \mathrm{hPa}$ until day +28 . The differences in zonal wind in the troposphere are in accordance with the 30-day mean PT500 employed for classification. It should be noted, however, that the differences emerge well before the key-day, that is, during the growth stage of the SSW event. The high-latitude tropospheric zonal winds are similar for the two types of events up to day -10. After this day, however, the wind in the cold composite strengthens while that in the warm composite weakens across the key-day. The respective anomalies are maintained to around day +30 . This result shows that the tropospheric anomalies are established during the growth stage of the SSW, one week prior to the key-day. 
The dynamics of this change in zonal wind are examined using the anomalies in the EP flux and the divergence from the average over days -10 to -1 , as shown in Figure 3. The enhanced upward EP fluxes into the stratosphere are evident in both composites, but clear differences can be seen in the troposphere. In the warm composite, waves propagate more poleward, while in the cold composite, waves propagate more equatorward. These divergent anomalies of the EP flux indicate that weak deceleration anomalies are generated in the high-latitude middle troposphere during warm events, while strong acceleration anomalies are produced in the high-latitude upper troposphere in cold events. The differences in wave forcing is mainly attributable to differences in planetary wave forcing. In both composites, upward propagation of the wavenumber-1 wave (wave-1) is enhanced, but the enhancement is stronger in the cold composite. For wave-2, upward propagation is enhanced in the warm composite and weakened in the cold composite. The upward propagation of wave-1 and wave-2 changes the tropospheric wave forcing, resulting in the generation of westerly (easterly) wind anomalies during the growth stage of SSW events in the cold (warm) composite. The time series of amplitude anomalies of geopotential height wave- 1 and wave- 2 at $60^{\circ} \mathrm{N}$, $100 \mathrm{hPa}$ are shown in Figures $2 \mathrm{~b}$ and 2c. The difference in geopotential height for wave-2 is particularly large during the growth stage. After the key-day, the tropospheric anomalies have been maintained until 4 weeks later (Figure 2a).

The horizontal structures in the troposphere are shown in Figure 4 as composites of geopotential height anomalies at $500 \mathrm{hPa}$. During the growth stage, negative anomalies appear over the Bering Sea in both composites. However, differences can be seen in the region from the North Atlantic Ocean to eastern Asia. In the warm composite, negative anomalies occur over England and eastern Asia, and positive anomalies appear over western Siberia. This pattern is reversed in the cold composite. This pattern of anomalies resembles the Eurasian Pattern, one of the teleconnection patterns discovered by Wallace and Gutzler [1981]. During the growth stage, the pattern is very similar to the EU1 Eurasian Pattern, which presents the leading eigenmode of wave activity flux [Plumb, 1985] over the Atlantic Ocean and Eurasian continent [Ohhashi and Yamazaki, 1999]. At three centers of action in the EU1 pattern, differences in anomalies are 
statistically significant. Wave activity associated with the EU1 pattern acts to generate the tropospheric Arctic Oscillation [Ohhashi and Yamazaki, 1999], forcing the negative phase of the AO after SSW in warm events (Figure 4, lower left), and a weak positive AO phase during cold events (Figure 4, lower right). After the SSW events, statistically significant patterns of alternating sign appear over the Arctic Ocean and northwestern the Atlantic Ocean.

\section{Discussion and conclusions}

The SSW events that propagate into the troposphere are associated with the strong wave-2 and wave-1 upward EP flux. The SSW events that do not propagate into the troposphere, that is, the events that induce polar tropospheric cooling, are associated with the weakened wave-2 and enhanced wave-1 upward EP flux. This finding is consistent with the results of AGCM simulations [Yoden et al., 1999], although the signals in the simulations do not propagate to the surface.

The tropospheric anomalies observed in this study are generated during the SSW growth stage at a time when planetary waves strengthen in the troposphere and propagate into the stratosphere. The EP flux propagates upward and poleward in warm events, and upward and equatorward in cold events. This difference in wave propagation causes a difference in wave forcing in the troposphere. In the stratosphere above $100 \mathrm{hPa}$, wave forcing is similar in both types of SSW event, commonly inducing anomalies of strong downward residual circulation in the polar regions above $150 \mathrm{hPa}$. Stratospheric wave forcing, in principle, produces downward motion in the polar troposphere [Haynes et al., 1991]. However, the residual circulation anomalies induced in the troposphere differ markedly between the two types of event. The strong downward residual motion penetrates to the surface in warm events, whereas tropospheric circulation in an opposite sense to that in the stratosphere is formed during cold events due to tropospheric wave forcing, which overcomes the stratospheric forcing before the key-day. Planetary waves (wave-1 and wave-2) contribute largely to this tropospheric forcing during the growth stage. In the troposphere, the Eurasian pattern during the growth stage differs substantially between the two. 
We categorized the SSW events by 30-day mean polar temperature at $500 \mathrm{hPa}$ after the key-day and found the behaviors of planetary waves, especially wave-2, are different between two cases. It suggests that the warming types can tell whether the downward propagation occurs or not. Pure wave- 1 warming represents displacement of the polar vortex, while wave-2 warming occurs by splitting of the vortex. Subjective inspection of each SSW event at $50 \mathrm{hPa}$ reveals that 14 of the events are splitting events, while the remaining 37 events are displacement ones. Among the 14 splitting events, 11 were followed by tropospheric polar warming and 3 by cooling. Among the 37 displacement events, 17 were followed by tropospheric polar warming and 20 by cooling. For example, the December 1998 SSW event that did not propagate into the troposphere was a displacement (wave-1) event, whereas the subsequent SSW in February 1999 that propagated into the troposphere was a splitting (wave-2) event. A splitting SSW is therefore likely to be followed by a negative phase of AO in the troposphere.

To confirm this more objectively, the SSW events are classified by upward wave-2 EP flux at $150 \mathrm{hPa}, 50-80^{\circ} \mathrm{N}$, average for day -7 to -1 . Figure 5 shows the high-latitude zonal mean zonal wind anomalies for the above-normal wave-2 EP flux composite (16 events) and the below-normal composite (35 events). In the enhanced wave-2 composite, the tropospheric negative anomalies appear around day -10, while the negative anomalies appear around day +10 in the reduced wave- 2 composite. Before the key-day, the zonal winds are decelerated in the enhanced wave- 2 composite and accelerated in the reduced wave- 2 composite, which are forced by planetary-wave forcings. The differences around the key-day in the troposphere and lower stratosphere are statistically significant. After day +10 , the enhanced wave- 2 composite shows larger negative anomalies and the reduced composite shows smaller negative anomalies, though the differences are not statistically significant.

The present results do not contradict the influence of stratospheric processes on the troposphere. In all SSW events, anomalies in high-latitude zonal wind propagate downward (not shown), as found in previous studies [Baldwin and Dunkerton, 1999, 2001; Limpasuvan et al., 2004]. Negative anomalies propagate into the troposphere one 
week after the SSW and persist until day +55 . Even in Figure 5, shortly after the key-day, both composites show the decelerations. This may be an indication of the stratospheric forcing on the troposphere through dynamical stratospheric wave forcing around day +10 , followed by radiative forcing afterward [Thompson et al., 2005].

The present study distinguished two types of SSW: a wave-2 type SSW event and a wave-1 type SSW event. Consideration of this distinction between types of SSW can be expected to improve the accuracy of long-range weather forecasting.

\section{Acknowledgments}

We thank Y. Kuroda, anonymous reviewers and staff members of the graduate school for valuable comments.

\section{References}

Andrews, D. G., J. R. Holton, and C. B. Leovy, "Middle Atmosphere Dynamics", Academic Press, 489pp, 1987.

Baldwin, M. P., and T. J. Dunkerton, Propagation of the Arctic Oscillation from the stratosphere to the troposphere. J. Geophys. Res., 104, 30937-30946, 1999.

Baldwin, M. P., and T. J. Dunkerton, Stratospheric harbingers of anomalous weather regimes, Science, 294, 581-584, 2001.

Haynes, P. H., C. J. Marks, M. E. McIntyre, T. G. Shepherd and K. P. Shine, On the downward control of extratropical diabatic circulations by eddy-induced mean zonal forces. J. Atmos. Sci., 48, 651-679, 1991.

Limpasuvan, V., D. W. J. Thompson, and D. L. Hartmann, The life cycle of the Northern Hemisphere sudden stratospheric warmings, J. Climate, 17, 2584-2596, 2004.

Ohhashi, Y., and K. yamazaki, Variability of the Eurasian pattern and its interpretation by wave activity flux. J. Meteor. Soc. Japan, 77, 495-511, 1999.

Plumb, R. A., On the three-dimensional propagation of stationary waves. J. Atmos. Sci., 42, 217-229, 1985.

Simmons, A. J., and J. K. Gibson, The ERA-40 project plan, ERA-40 Proj. Rep. Ser. 1, 63 pp., Eur. Cent. For Medium Range Weather Forecasts, Reading, UK, 2000. 
Thompson, D. W. J., and J. M. Wallace, The Arctic Oscillation signature in the wintertime geopotential height and temperature fields. Geophys. Res. Lett., 25, 1297-1300, 1998.

Thompson, D. W. J., and J. M. Wallace, Annular modes in the extratropical circulation. Part I: Month-to-month variability. J. Climate, 13, 1000-1016, 2000.

Thompson, D. W. J., J. C. Furtado, and T. G. Shepherd, The "Direct Effect" of stratospheric variability on the troposphere. J. Atmos. Sci., 2005 (submitted).

Wallace, J. M., D. S. Gutzler, Teleconnections in the geopotential height field during the Northern Hemisphere winter, Mon. Wea. Rev., 109, 784-812, 1981.

Yoden, S., T. Yamaga, S. Pawson and U. Langematz, A composite analysis of the stratospheric sudden warmings simulated in a perpetual January integration of the Berlin TSM GCM, J. Meteor. Soc. Japan, 77(2), 431-445, 1999. 

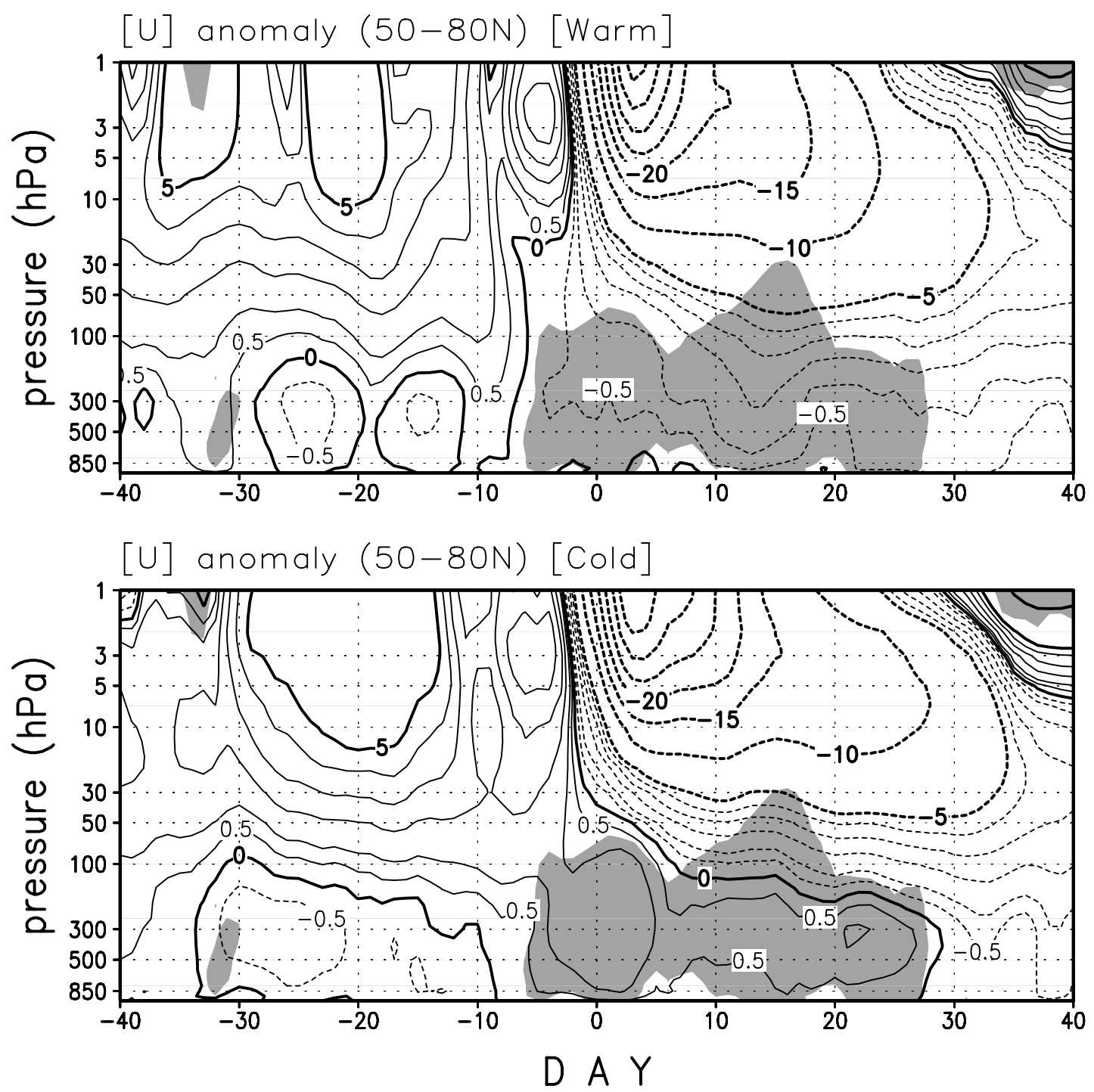

Figure 1 Time-height composite of zonal mean zonal wind anomalies averaged over $50-80^{\circ} \mathrm{N}$ for tropospheric warm events (top) and cold events (bottom). Abscissa denotes days from the key-day. The area of difference between tropospheric warm and cold events with statistical significance at the $95 \%$ level ( $t$-test) is shaded. 

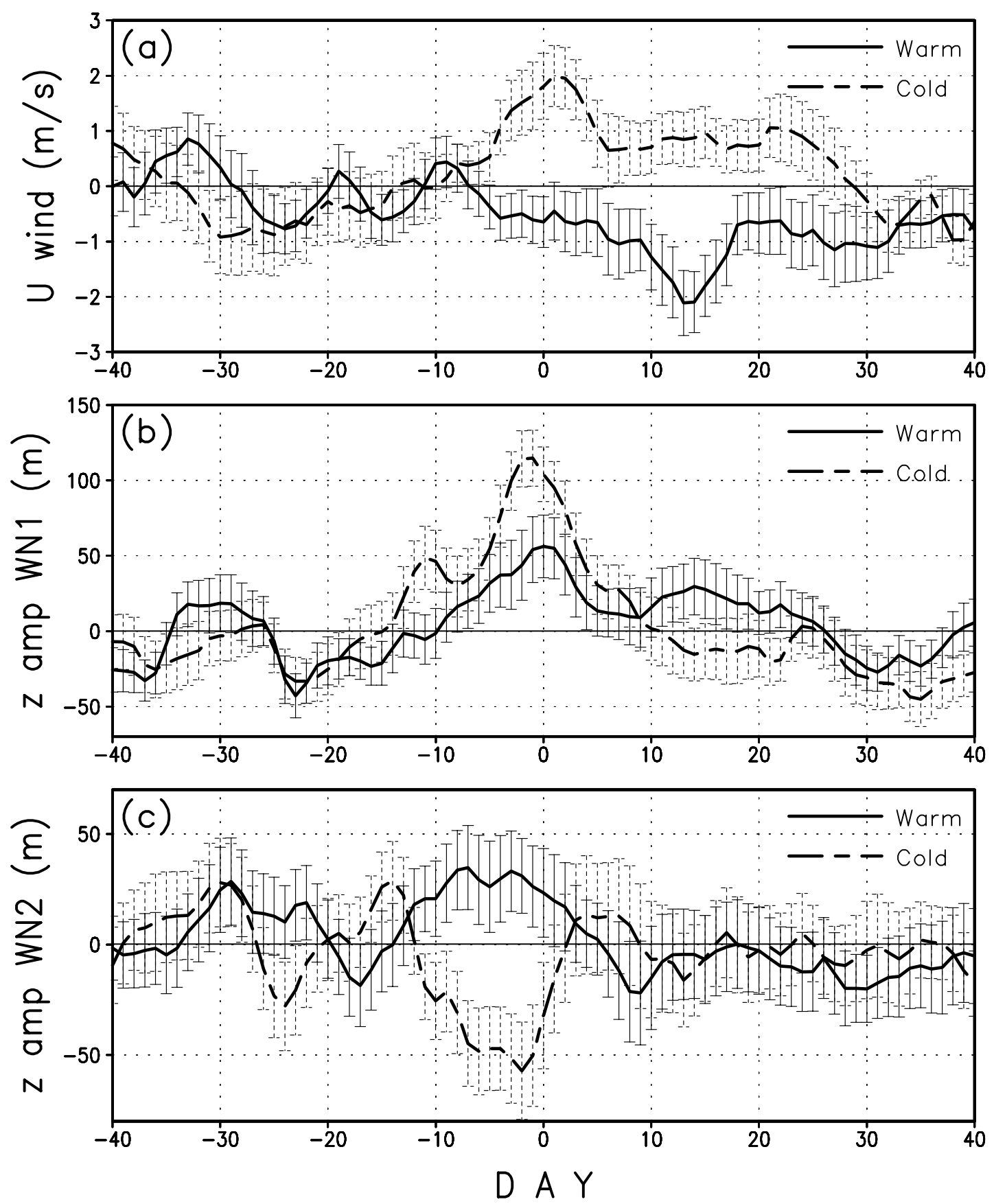

Figure 2: Evolution of anomalies in high-latitude $\left(50-80^{\circ} \mathrm{N}\right)$ zonal wind at $300 \mathrm{hPa}$ (a: top) and wave-1 (b: middle) and wave-2 (c: bottom) geopotential height at $60^{\circ} \mathrm{N}, 100 \mathrm{hPa}$ for warm (solid line) and cold (dashed line) tropospheric polar temperature composites. Error bars denote standard errors. 

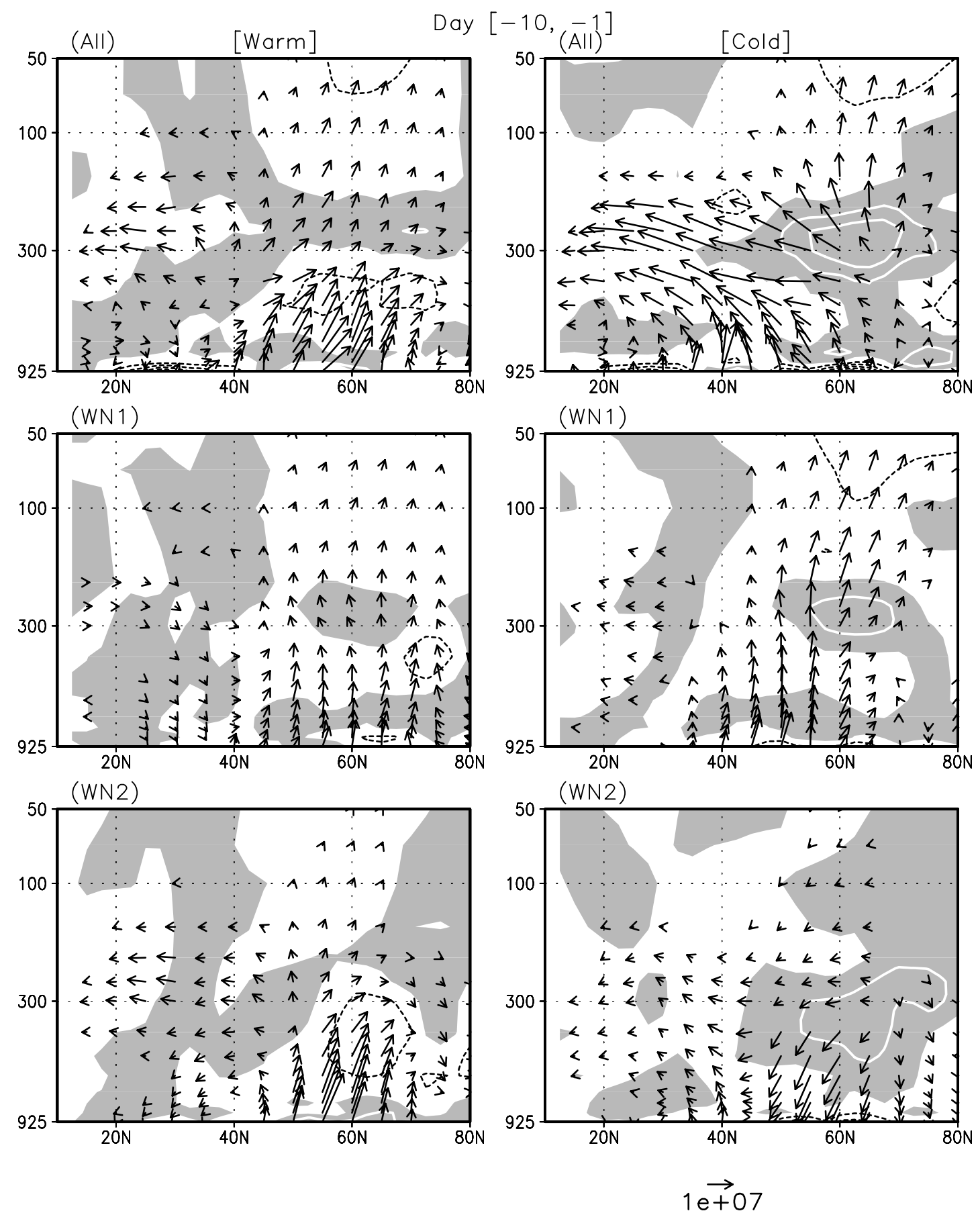

Figure 3: Anomalies in the EP flux and divergence (top), and wave-1 (middle) and wave-2 (bottom) components of the EP flux for warm (left) and cold (right) tropospheric polar temperature composites. A horizontal scale of arrow is shown at bottom right for $1 \times 10^{7} \mathrm{~kg} \mathrm{~s}^{-2}$. The vertical length of the arrow is 100 times larger than the horizontal. Contours denote the EP flux divergence (every $1 \mathrm{~m} \mathrm{~s}^{-1}$ day $^{-1}$ ). 


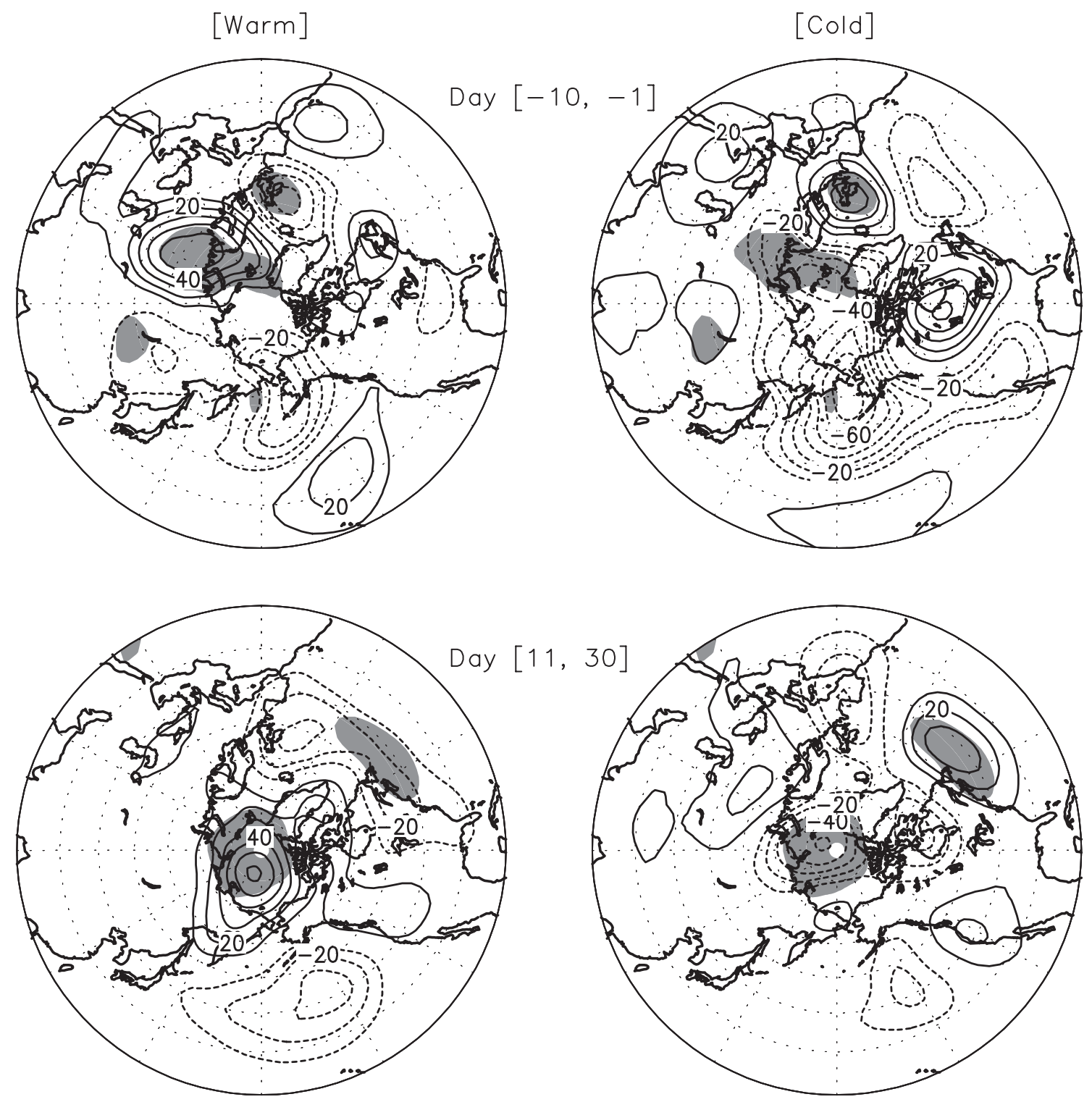

Figure 4: Anomalies in geopotential height at $500 \mathrm{hPa}$ averaged from day -10 to day -1 (top) and day +11 to day +30 (bottom) for warm (left) and cold (right) tropospheric polar temperature composites. Contour interval is $10 \mathrm{gpm}$ (zero contours are omitted). The area of difference between tropospheric warm and cold events with statistical significance at the 95\% level ( $t$-test) is shaded. 

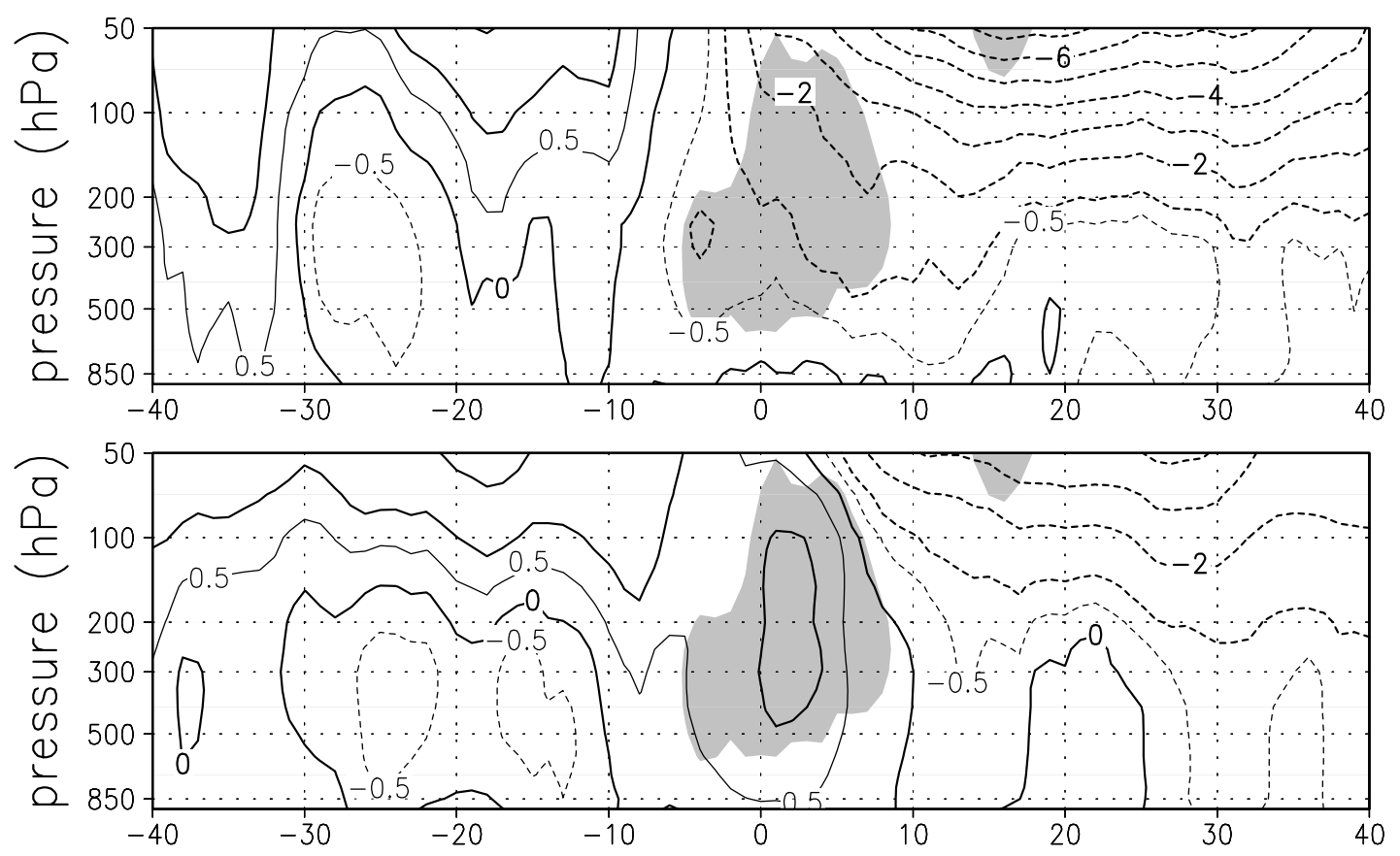

Figure 5: Same as in Figure 1 except for classified by above-normal (top) and below-normal (bottom) upward wave-2 EP flux at $150 \mathrm{hPa}, 50-80^{\circ} \mathrm{N}$ averaged for day -7 to -1 . 